\title{
Etude comparée de l'activité enzymatique de 3 variétés d'artichauts du genre Cinara Cardunculus $L$ sur la coagulation du lait
}

\author{
par \\ J. TSOULI \\ Département de Biologie Appliquée \\ Institut Universitaire de Technologie 1 \\ 43, boulevard du 11 novembre 1918 - 69621 Villeurbanne
}

Dans une étude antérieure [1], on a montré qu'une solution extraite des fleurs d'artichauts sauvages (genre Cinara Cardunculus $L$ ) dans $\mathrm{Nacl}$ à 0,9 p. 100 à $+18^{\circ} \mathrm{C}$, avait sur le lait un pouvoir coagulant plus important que celui d'une solution identique préparée dans $\mathrm{H}_{2} \mathrm{O}$.

Aussi, a-t-on entrepris dans le présent travail, l'étude de l'influence du taux de $\mathrm{NaCl}$ et de la température sur le rendement d'extraction de l'activité enzymatique de l'artichaut. En outre, il a paru important de comparer, entre elles, les activités de différentes variétés d'artichauts afin de trouver le végétal qui donne le meilleur rendement et de localiser dans la plante la partie la plus riche en activité enzymatique.

Cette étude a été réalisée en novembre 1971 sur des échantillons d'artichauts : Gros Camus de Bretagne, Violets hâtifs de Provence et sauvages, tous du genre Cinara Cardunculus $L$ en provenant de la récolte de juillet 1969 .

Le travail expérimental est le suivant :

\section{I. - LOCALISATION DU SYSTEME ENZYMATIQUE DANS LE VEGETAL}

On a étudié la distribution de l'enzyme dans le végétal. Pour cela, on a réduit en poudre pédoncule, réceptacle et bractées de l'artichaut et on a testé leur activité enzymatique dans les mêmes conditions que pour les fleurs.

Les solutions ainsi obtenues avec les artichauts bretons, violets et sauvages ne coagulent pas le lait au bout d'un temps $>2 \mathrm{~h}$. Ce qui confirme les observations faites par Christen et al. sur le 
chardon d'Espagne [2]. Cependant, quand on effectue le même travail sur l'artichaut vert de Provence, on enregistre une certaine activité enzymatique au niveau du réceptacle et du pédoncule (un volume d'une solution extraite à partir de $25 \mathrm{~g}$ de poudre de réceptacle ou de pédoncule/ 1 coagule 10 volumes de lait à $40^{\circ} \mathrm{C}$ au bout de $5 \mathrm{~h}$ et $3 \mathrm{~h}$ respectivement).

Cette variété est d'ailleurs récoltée à une période où les fleurs sont très jeunes et pratiquement confondues avec le réceptacle à l'endroit de leur insertion ce qui explique le fait que sur le plan alimentaire, l'artichaut vert est presque entièrement consommé.

Il faut donc attendre le stade de la floraison si l'on veut améliorer le rendement et obtenir une activité enzymatique optimum.

\section{II. - EXTRAITS ENZYMATIQUES OBTENUS DES FLEURS D'ARTICHAUTS ET TECHNIQUES EXPERIMENTALES}

Les extractions sont effectuées comme précédemment [1] aux différences suivantes près :

- On fait varier la température d'extraction $\left(+23^{\circ} \mathrm{C}\right.$ et $\left.+4^{\circ} \mathrm{C}\right)$, la durée d'extraction ( $8 \mathrm{j}$ au lieu de $24 \mathrm{~h}$ ) et le taux de $\mathrm{NaCl}$ des solutions ( 5 p. 100 à 0,5 p. 100); il y a lieu cependant d'être attentif au développement des moisissures à la surface des solutions pendant la durée d'extraction à $+23^{\circ} \mathrm{C}$.

- Pour la mesure de l'activité enzymatique, on a utilisé la notion de "force " définie par Soxhlet $[3,4]$ et qui est normalement employée. Cette «force " représente le nombre de volume de lait frais de mélange coagulé par un volume de présure en $40 \mathrm{mn}$ à $35^{\circ} \mathrm{C}$. Ainsi, si l'on prend un volume v de présure ou d'extrait, un volume V de lait et on mesure un temps de coagulation $\mathrm{T}$ en secondes, on calcule :

$$
F=\frac{2400 \mathrm{~V}}{\mathrm{TV}}
$$

Dans le présent travail, cette définition a été appliquée au lait entier en poudre du commerce qui est homogène et régulier.

Après reconstitution, ce lait a un taux de matière grasse de $34 \mathrm{~g} / \mathrm{l}$, un $\mathrm{pH}$ de $6,4-6,5$ et une acidité de $16^{\circ}$ Dornic (1 ${ }^{\circ}$ Dornic correspondant à $1 \mathrm{dg}$ d'acide lactique par litre de lait).

Pour les autres opérations, ce lait reconstitué est additionné

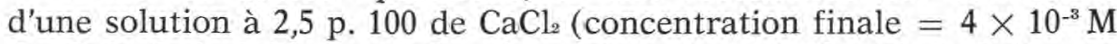
de $\mathrm{CaCl}_{2} / 1$ de lait). Les tubes à essai contenant $10 \mathrm{~cm}^{3}$ de lait sont placés dans un thermostat à $35^{\circ} \mathrm{C}$ et après équilibration thermique, on leur ajoute $1 \mathrm{~cm}^{3}$ de la solution enzymatique d'artichaut (40 $\mathrm{g}$ de fleurs d'artichauts à 94 p. 100 de matière sèche/1 de solution). Les 
valeurs trouvées représentent la moyenne d'au moins deux essais pour chaque variété d'artichauts.

Les résultats se trouvent résumés dans les tableaux 1 et 2 .

\section{RESULTATS ET DISCUSSION}

Le tableau 1 montre que le taux d'extraction de l'enzyme n'est pas modifié par le taux de concentration en $\mathrm{NaCl}$, ce qui est vérifié également par les résultats du tableau 2.

\section{TABLEAU 1}

Activité enzymatique résultant des extraits de fleurs d'artichauts dans $\mathrm{NaCl} 0,8$ et 3 p. 100

\begin{tabular}{|c|c|c|}
\hline $\begin{array}{c}\text { Origine de l'enzyme } \\
\text { et } \\
\text { conditions d'extraction }\end{array}$ & $\begin{array}{c}\text { Temps } \\
\text { de coagulation } \\
\text { en secondes } \\
\text { à } 35^{\circ} \mathrm{C}\end{array}$ & $\begin{array}{l}\text { Force } \quad 2400 \times V \\
\text { S }=\frac{T V}{\text { Soxhlet }}\end{array}$ \\
\hline 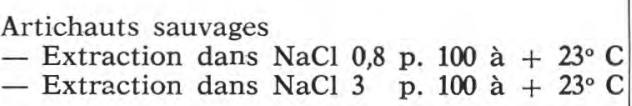 & $\begin{array}{l}537 \\
520\end{array}$ & $\begin{array}{l}44,7 \\
46\end{array}$ \\
\hline 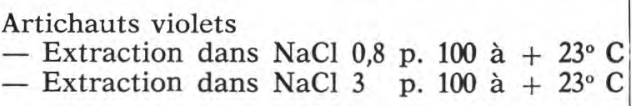 & $\begin{array}{r}1200 \\
800\end{array}$ & $\begin{array}{l}20 \\
30\end{array}$ \\
\hline 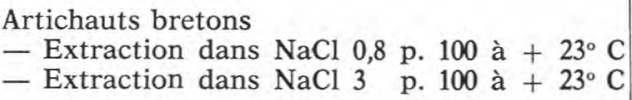 & $\begin{array}{l}1320 \\
1380\end{array}$ & $\begin{array}{l}18 \\
17\end{array}$ \\
\hline
\end{tabular}

Les valeurs relatives aux concentrations 0,8 et 3 p. $100 \mathrm{NaCl}$ (tab. 1) peuvent donc être considérées comme représentatives des autres solutions. On note également que l'artichaut sauvage a une activité enzymatique plus importante, l'artichaut violet vient ensuite et que l'artichaut breton, dans les conditions actuelles, ne présente qu'un intérêt secondaire.

Là aussi, il faut tenir compte du stade végétatif ; en effet le stade floral de l'artichaut violet hâtif de Provence était bien plus avancé que les autres et c'est avec lui (ainsi qu'avec l'artichaut sauvage) que l'on obtient le coagulum le plus ferme. 
TABLEAU 2 (première partie)

Activité enzymatique des solutions : durée de coagulation en $\mathrm{mn}$ et $\mathrm{R}=\frac{\text { Volume de présure }}{\text { Volumes de lait coagulé }}$

\begin{tabular}{|c|c|c|c|c|c|c|c|c|c|c|c|}
\hline $\begin{array}{c}\text { Origine de l'enzyme } \\
\text { et } \\
\text { conditions d'extraction }\end{array}$ & $\begin{array}{l}\text { Concentration } \\
\text { des solutions } \\
\text { en } \mathrm{NaCl}\end{array}$ & $5 \%$ & $1 / \mathrm{R}$ & $4 \%$ & $1 / \mathrm{R}$ & $3 \%$ & $1 / \mathrm{R}$ & $2 \%$ & $1 / \mathrm{R}$ & $1 \%$ & $1 / \mathrm{R}$ \\
\hline Artichauts sauvages & $\begin{array}{r}23^{\circ} \mathrm{C} \\
4^{\circ} \mathrm{C}\end{array}$ & $\begin{array}{c}3,66 \\
5,25\end{array}$ & $\begin{array}{r}109 \\
76\end{array}$ & $\begin{array}{l}3,66 \\
4,66\end{array}$ & $\begin{array}{c}109 \\
85,8\end{array}$ & $\begin{array}{l}3,5 \\
4,66\end{array}$ & $\begin{array}{c}114 \\
85,8\end{array}$ & $\begin{array}{l}3,33 \\
4\end{array}$ & $\begin{array}{l}120 \\
100\end{array}$ & $\begin{array}{l}3,25 \\
3,8\end{array}$ & $\begin{array}{l}123 \\
105\end{array}$ \\
\hline Artichauts Violets hâtifs de Provence & $\begin{array}{r}23^{\circ} \mathrm{C} \\
4^{\circ} \mathrm{C}\end{array}$ & $\begin{array}{c}8,8 \\
12,75\end{array}$ & $\begin{array}{l}45,5 \\
31,3\end{array}$ & $\begin{array}{l}8 \\
8,33\end{array}$ & $\begin{array}{l}50 \\
48\end{array}$ & $\begin{array}{l}6,8 \\
8,66\end{array}$ & $\begin{array}{l}58,8 \\
46\end{array}$ & $\begin{array}{l}6,8 \\
8,33\end{array}$ & $\begin{array}{l}58,8 \\
48\end{array}$ & $\begin{array}{l}6,8 \\
9,8\end{array}$ & $\begin{array}{l}58,8 \\
40,8\end{array}$ \\
\hline Artichauts bretons & $\begin{array}{r}23^{\circ} \mathrm{C} \\
4^{\circ} \mathrm{C}\end{array}$ & $\begin{array}{l}15 \\
16,16\end{array}$ & $\begin{array}{l}26,6 \\
24,7\end{array}$ & \begin{tabular}{|l}
12,8 \\
15,75
\end{tabular} & $\begin{array}{l}31,2 \\
25,4\end{array}$ & $\begin{array}{l}12,8 \\
16\end{array}$ & $\begin{array}{l}31,2 \\
25\end{array}$ & $\begin{array}{l}10 \\
20\end{array}$ & $\begin{array}{l}40 \\
20\end{array}$ & $\begin{array}{l}12 \\
19\end{array}$ & $\begin{array}{l}33,3 \\
21\end{array}$ \\
\hline
\end{tabular}




\section{TABLEAU 2 (deuxième partie)}

Activité enzymatique des solutions : durée de coagulation en $\mathrm{mn}$ et $\mathrm{R}=\frac{\text { Volume de présure }}{\text { Volumes de lait coagulé }}$

\begin{tabular}{|c|c|c|c|c|c|c|c|c|c|c|c|}
\hline $\begin{array}{c}\text { Origine de l'enzyme } \\
\text { et } \\
\text { conditions d'extraction }\end{array}$ & $\begin{array}{l}\text { Concentration } \\
\text { des solutions } \\
\text { en } \mathrm{NaCl}\end{array}$ & $0,9 \%$ & $1 / \mathrm{R}$ & $0,8 \%$ & $1 / R$ & $0,7 \%$ & $1 / R$ & $0,6 \%$ & $1 / \mathrm{R}$ & $0,5 \%$ & $1 / \mathrm{R}$ \\
\hline Artichauts sauvages & $\begin{array}{r}23^{\circ} \mathrm{C} \\
4^{\circ} \mathrm{C}\end{array}$ & $\begin{array}{l}4,25 \\
4,5\end{array}$ & $\begin{array}{l}94 \\
88\end{array}$ & $\begin{array}{l}3,25 \\
3,5\end{array}$ & $\begin{array}{l}123 \\
114\end{array}$ & $\begin{array}{l}3,5 \\
6,4\end{array}$ & $\begin{array}{r}114 \\
62\end{array}$ & $\begin{array}{l}6 \\
3,5\end{array}$ & $\begin{array}{r}66 \\
114\end{array}$ & $\begin{array}{l}4,5 \\
4,5\end{array}$ & $\begin{array}{l}88 \\
88\end{array}$ \\
\hline Artichauts Violets hâtifs de Provence & $\begin{array}{r}23^{\circ} \mathrm{C} \\
4^{\circ} \mathrm{C}\end{array}$ & $\begin{array}{l}6,8 \\
7,16\end{array}$ & $\begin{array}{l}58,8 \\
55,8\end{array}$ & $\begin{array}{l}6,66 \\
9\end{array}$ & $\begin{array}{l}60 \\
44,5\end{array}$ & $\begin{array}{r}7 \\
15\end{array}$ & $\begin{array}{l}57 \\
26,6\end{array}$ & $\begin{array}{l}7,75 \\
6,5\end{array}$ & $\begin{array}{l}51,6 \\
61,5\end{array}$ & $\begin{array}{l}6,5 \\
8,75\end{array}$ & $\begin{array}{l}61,5 \\
45,7\end{array}$ \\
\hline Artichauts bretons & $\begin{array}{r}23^{\circ} \mathrm{C} \\
4^{\circ} \mathrm{C}\end{array}$ & $\begin{array}{l}14,5 \\
16\end{array}$ & $\begin{array}{l}27,5 \\
25\end{array}$ & $\begin{array}{l}11,8 \\
15,25\end{array}$ & $\begin{array}{l}33,9 \\
26,2\end{array}$ & $\begin{array}{l}11,8 \\
15,8\end{array}$ & $\begin{array}{l}33,9 \\
25,3\end{array}$ & $\begin{array}{l}11,75 \\
17\end{array}$ & $\begin{array}{l}34 \\
23,5\end{array}$ & $\begin{array}{l}16,5 \\
21\end{array}$ & $\begin{array}{l}24,2 \\
19\end{array}$ \\
\hline
\end{tabular}


Si l'on regarde le tableau 2, la hiérarchie de l'activité enzymatique des différentes variétés est respectée et l'on a :

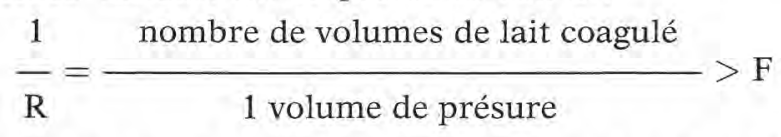

Cela résulte de l'addition au lait de $4 \times 10^{-3} \mathrm{M}$ de $\mathrm{CaCl}_{2} / 1$ qui favorise la coagulation $[3,5]$.

Quand on compare ces résultats à ceux obtenus antérieurement [1], l'augmentation de l'activité enzymatique, dans le cas présent, s'explique d'abord par le taux plus important de $\mathrm{CaCl}_{2}$ mais aussi par la durée d'extraction plus longue ( $8 \mathrm{j}$ au lieu de $24 \mathrm{~h}$ ) qui augmente la concentration en enzyme des solutions.

De plus, si le taux de $\mathrm{NaCl}$ n'a pas d'influence sur celui de l'extraction enzymatique, il reste cependant qu'une force ionique minimum est nécessaire pour une meilleure extraction puisqu'avec $\mathrm{H}_{2} \mathrm{O}$ seule, on obtient des solutions moins actives [1].

On note aussi qu'à $+23^{\circ} \mathrm{C}$, les solutions obtenues sont plus actives qu'à $+4^{\circ} \mathrm{C}$. Cela fait penser à l'existence d'une température optimum pour un meilleur rendement.

En conclusion, on retiendra pour les travaux ultérieurs, les artichauts sauvages et violets sans pour autant négliger les cas des artichauts bretons car étant donné les quantités plus importantes de matière première dont on peut disposer à bon marché, l'amélioration des conditions d'extraction et la concentration des solutions pourraient aboutir à des résultats intéressants.

\section{Rés u m é}

La coagulation du lait par des extraits de fleurs de trois variétés d'artichauts : sauvages, Gros Camus de Bretagne et Violets hâtifs de Provence, après $8 \mathrm{j}$ d'extraction à $+23^{\circ} \mathrm{C}$ et $+4^{\circ} \mathrm{C}$ dans différentes solutions de $\mathrm{NaCl}$, montre que les artichauts sauvages présentent une activité enzymatique plus importante que les deux autres.

Les extractions sur les autres parties du végétal montrent que toute l'activité enzymatique se retrouve essentiellement dans les fleurs.

De plus, des solutions ayant des concentrations croissantes de $\mathrm{NaCl}$ ne modifient pas le taux d'extraction ; en revanche, la température joue un rôle prépondérant : à $+23^{\circ} \mathrm{C}$, les solutions obtenues sont plus actives qu'à $+4^{\circ} \mathrm{C}$. 


\section{S u m m a r y}

Milk is clotted by the extracts of flowers from three different artichokes : a wild type a gros Camus from Brittany and a violet hatif from Provence.

8 days'extractions at $+23^{\circ} \mathrm{C}$ and at $+4^{\circ} \mathrm{C}$ in different solutions of $\mathrm{NaCl}$ shows that the wild artichokes present an enzymatic activity which is greater than the two others.

Extractions made with the other parts of the artichokes show that all the enzymatic activity is essentially localised in the flowers.

Moreover solutions with increased concentrations of $\mathrm{NaCl}$ do not modify the rate of extraction; on the other hand the temperature plays a preponderant role since at $23^{\circ} \mathrm{C}$ the solutions obtained are more active than at $+4^{\circ} \mathrm{C}$.

Reçu pour publication le 20 avril 1974

\section{Bibliographie}

[1] Tsouli (1970). - Cr. Ac. Sci., t. 270, 369-399, janvier.

[2] Christen et coll. (1935). - Le Lait, 144-145, 354-363.

[3] Alais (1965). - Ed. Sep., Paris.

[4] Veisseyre (1966). - Maison Rustique, Paris, 379.

[5] Garnier et coll. (1968). - Ann. Nutri. Alim., 22, B 495 - B 452. 